\title{
Avaliação do Estado de Saúde pelo Questionário de Angina de Seattle em Pacientes com Síndrome Coronária Aguda
}

\author{
Alexandre Schaan de Quadros', Emiliane Nogueira de Souza', Rubia Maestri', Camila Albarran', \\ Carlos A. M. Gottschall'1', Rogério Sarmento-Leite ${ }^{1}$
}

\section{RESUMO}

Introdução: Estudos que analisaram o estado de saúde não avaliaram diferenças entre pacientes com angina instável e infarto agudo do miocárdio com (IAMCST) e sem (IAMSST) elevação do segmento ST. Este estudo teve como objetivo comparar índices do estado de saúde, de acordo com o Questionário de Angina de Seattle (QAS), em pacientes com síndrome coronária aguda (SCA). Métodos: O QAS foi aplicado na admissão hospitalar e aos seis meses de acompanhamento em 391 pacientes com SCA. Foram analisados três domínios: limitação física (D1), frequência da angina (D3) e qualidade de vida (D5). Índices maiores em D1, D3 e D5 significam menor limitação física, menor frequência da angina e melhor qualidade de vida, respectivamente. Resultados: Na internação hospitalar, pacientes com angina instável apresentaram piores índices de frequência da angina e de qualidade de vida que os portadores de IAMSST, e piores índices de atividade física, frequência da angina e qualidade de vida que aqueles com IAMCST. Aos seis meses de seguimento, os pacientes com angina instável ainda apresentaram piores índices de atividade física, frequência da angina e qualidade de vida que os portadores de IAMCST, mas sem diferença significativa em relação aos pacientes com IAMSST. Pacientes com angina instável foram aqueles que apresentaram maior ganho em todos os domínios do QAS. Conclusões: No espectro da SCA, os pacientes com angina instável demonstraram pior estado de saúde, tanto na internação hospitalar como aos seis meses de acompanhamento, mas, por outro lado, foram os que apresentaram o maior ganho em cada um dos domínios avaliados pelo QAS.

DESCRITORES: Síndrome coronariana aguda. Qualidade de vida. Nível de saúde. Angioplastia coronária com balão.

\section{ABSTRACT}

\section{Health Status Assessment by the Seattle Angina Questionnaire in Acute Coronary Syndromes}

Background: Studies assessing health status have not addressed differences among patients with unstable angina, nonST elevation myocardial infarction (NSTEMI) and ST-elevation myocardial infarction (STEMI). This study was aimed at comparing health status scores according to the Seattle Angina Questionnaire (SAQ) in patients with acute coronary syndromes (ACS). Methods: SAQ was applied at hospital admission and at 6-month follow-up in 391 patients with ACS. Three domains were analyzed: physical limitation (D1), anginal frequency (D3) and disease perception (D5). Higher D1, D3 and D5 scores indicated less physical limitation, lower frequency of angina and better quality of life, respectively. Results: At hospital admission, patients with unstable angina presented significantly lower scores of anginal frequency and disease perception than those with NSTEMI, and significantly lower scores of physical activity, anginal frequency and disease perception than those with STEMI. At the 6-month follow-up, patients with unstable angina still presented lower scores of physical activity, anginal frequency and disease perception than those with STEMI, but there were no significant differences when compared to those with NSTEMI. The SAQ domains increased to a greater extent in patients with unstable angina. Conclusions: Patients with unstable angina had worse health status at hospital admission and at the 6-month follow-up but, on the other hand, showed the largest improvements for each one of the SAQ domains analyzed.

KEY-WORDS: Acute coronary syndrome. Quality of life. Health status. Angioplasty, balloon, coronary.

\footnotetext{
1 Instituto de Cardiologia do Rio Grande do Sul - Fundação Universitária de Cardiologia (IC-FUC) - Porto Alegre, RS, Brasil. Correspondência: Alexandre Schaan de Quadros. Av. Princesa Isabel, 395 - Santana - Porto Alegre, RS, Brasil - CEP 90620-000 E-mail: alesq@terra.com.br

Recebido em: 7/1/2011 • Aceito em: 10/3/2011
} 
0 estado de saúde inclui avaliações dos sintomas, função e qualidade de vida dos pacientes, e sua evolução é importante para os pacientes e para a sociedade. ${ }^{1,2}$ O Questionário de Angina de Seattle (QAS) foi proposto como uma ferramenta sensível e específica para avaliar o estado de saúde de pacientes com doença arterial coronária (DAC) ${ }^{3}$, sendo geralmente considerado mais sensível a pequenas alterações clínicas que os instrumentos genéricos. ${ }^{4,5}$ A maioria dos estudos reportados até a presente data tem incluído predominantemente pacientes estáveis, explorando as diferenças na medida do estado de saúde, com diferentes estratégias de tratamento, e identificando preditores de melhora. ${ }^{6-12}$

De acordo com orientação do American College of Cardiology/American Heart Association e da European Society of Cardiology, angina instável e infarto do miocárdio sem elevação do segmento ST (IAMSST) são consideradas condições intimamente relacionadas, e têm sido definidas como síndrome coronária aguda (SCA) sem elevação do segmento ST, no que concerne à estratificação do risco e às estratégias de tratamento. ${ }^{13,14}$

O QAS é uma ferramenta válida para avaliar alterações na frequência da angina, estabilidade e qualidade de vida relacionada à saúde, após revascularização miocárdica ou tratamento clínico medicamentoso em pacientes portadores de SCA. Entretanto, estudos prévios que analisaram o estado de saúde em pacientes com SCA não abordaram diferenças potenciais entre pacientes com angina instável ou com IAMSST. ${ }^{15-19}$ Além disso, há também escassos dados disponíveis sobre índices do QAS de pacientes com infarto do miocárdio com elevação do segmento ST (IAMCST). ${ }^{19,20}$

No presente estudo, foi testada a hipótese de que pacientes com angina instável ou IAMSST possam apresentar diferenças significativas do estado de saúde e de que a investigação com o QAS pode ajudar a entender melhor cada uma dessas condições clínicas. O objetivo deste estudo foi comparar índices do estado da saúde basal e após seis meses, avaliados pelo QAS, num grupo prospectivo e não selecionado de pacientes com SCA.

\section{MÉTODOS}

\section{Pacientes}

Os pacientes incluídos neste estudo foram os admitidos na emergência de um centro cardiológico de referência com diagnóstico de SCA durante o período compreendido entre janeiro e junho de 2004. Foram excluídos do presente estudo os portadores de doença neurológica ou psiquiátrica incapacitante, câncer, gravidez e choque cardiogênico e os incluídos em ensaios clínicos randomizados.

Foram prospectivamente registradas as características clínicas e angiográficas da população, as medica- ções usadas durante a hospitalização e prescritas no momento da alta hospitalar, bem como a evolução intra-hospitalar e a longo prazo, e incluídos em um banco de dados dedicado. O QAS foi aplicado nas primeiras 24 horas da admissão hospitalar e repetido após seis meses de seguimento, por um dos investigadores do estudo.

O estudo foi aprovado por um comitê institucional apropriado e todos os participantes assinaram um Termo de Consentimento Livre e Esclarecido.

\section{Apresentação clínica}

$\mathrm{Na}$ entrada, todos os pacientes foram diagnosticados como SCA, caracterizada como angina instável, IAMSST ou IAMCST. A angina instável foi definida como dor torácica típica, com duração superior a cinco minutos, iniciada nas últimas 96 horas antes da admissão hospitalar, com inversão de onda T ou depressão do segmento ST no eletrocardiograma, mas sem elevação de biomarcadores séricos de lesão miocárdica. IAMSST foi definido como dor torácica típica associada a inversão de onda T ou depressão do segmento ST no eletrocardiograma, e aumento de biomarcadores séricos de lesão miocárdica. IAMCST foi definido como dor típica prolongada e elevação do segmento ST, com indicação imediata de revascularização percutânea ou trombólise.

\section{Questionário de Angina de Seattle}

O QAS é um questionário específico para avaliar pacientes com DAC. ${ }^{3}$ No presente estudo foram analisados três domínios: limitação física (D1), frequência da angina (D3) e qualidade de vida (D5). O primeiro domínio utiliza perguntas para avaliar a capacidade de realizar atividades da vida diária e as perguntas incluídas em D3 analisam a frequência da angina e sua periodicidade. As perguntas em D5 avaliam o impacto do diagnóstico de DAC na qualidade de vida dos pacientes.

De acordo com as respostas dos pacientes, cada domínio recebe índices que variam de 0 a 100. Os pontos designados são relacionados ao estado de funcionalidade de cada domínio. Índices maiores em D1 e D3 significam menor limitação física e menor frequência da angina, respectivamente, e índices maiores em D5 significam melhor qualidade de vida. A descrição completa do QAS está disponível na publicação original de Spertus et al. ${ }^{3}$

Neste estudo são descritos os índices basais e após seis meses para cada domínio, de acordo com a SCA clínica apresentada. As diferenças entre as médias aos seis meses e basais representaram a variação em cada domínio.

\section{Seguimento e desfechos do estudo}

O seguimento clínico de seis meses foi realizado por avaliação clínica no ambulatório ou por contato 
telefônico. As incidências de angina, morte cardíaca, infarto do miocárdio ou procedimento de revascularização no período intra-hospitalar e em seis meses foram registradas.

\section{Análise estatística}

Foram realizadas análises estatísticas usando SPSS 11 (SPSS Inc., Chicago, Estados Unidos). As variáveis categóricas foram expressas como porcentuais e as variáveis contínuas, como média \pm desvio padrão. As diferenças entre os grupos de pacientes foram avaliadas pelo teste de qui-quadrado (variáveis categóricas) ou pelo teste ANOVA (variáveis contínuas). Para avaliar a interação entre o tempo (admissão e seguimento) e o grupo (angina instável, IAMSST e IAMCST) de cada domínio do QAS foi usado um delineamento de análise de variância com medidas repetidas.

Análise multivariada por regressão linear foi empregada para identificar preditores independentes de melhora dos índices do estado de saúde. ${ }^{21}$ As variáveis incluídas nesta análise foram raça, sexo, apresentação clínica da SCA, diabetes melito, hipertensão, infarto prévio do miocárdio e revascularização miocárdica.

\section{RESULTADOS}

Entre janeiro e junho de 2004, 501 pacientes foram encaminhados à emergência de nossa instituição com diagnóstico de SCA. Desses pacientes, 73 (15\%) foram excluídos pelos seguintes motivos: 13 pacientes com doenças neurológicas ou psiquiátricas incapacitantes; 8 pacientes com câncer; 2 pacientes grávidas; 7 pacientes com choque cardiogênico; e 43 pacientes incluídos em ensaios clínicos randomizados. Dos 428 pacientes incluídos no estudo, 12 morreram durante o período de seguimento e 25 pacientes não puderam ser contatados para o seguimento aos seis meses. A população final do estudo foi constituída por 391 pacientes.

As características basais da população de pacientes estão descritas na Tabela 1. Os pacientes com angina instável apresentaram perfil clínico mais complexo, com maior prevalência de sexo feminino, diabetes melito, insuficiência cardíaca, infarto do miocárdio e intervenções coronárias percutâneas (ICPs) prévias. O hábito de realizar exercício físico previamente à internação, o índice de massa corporal e os níveis de pressão arterial à admissão foram semelhantes nos três grupos de pacientes. Os picos de creatina quinase fração $M B(C K-M B)$ foram, respectivamente, de 44,9 mg/dl, 19,2 mg/dl e 4,6 mg/dl nos portadores de IAMCST, IAMSST e angina instável.

Em relação aos aspectos do tratamento, 90\% dos pacientes foram tratados com ácido acetilsalicílico (AAS), anticoagulantes e betabloqueadores na chegada ao hospital. Apesar da maior complexidade clínica, os pacientes com angina instável demonstraram menor uso de estatinas e de inibidores da enzima conversora de angiotensina, tanto na admissão como na alta hospitalar (Tabela 2).

Nos pacientes com IAMCST, o tratamento de reperfusão consistiu primariamente de ICP (77\%), 20\% foram tratados com trombolíticos e 2,5\% foram encaminhados para cirurgia. ICP foi usada em $21 \%$ dos portadores de angina instável e em $24 \%$ dos portadores de IAMSST e cirurgia cardíaca foi realizada em 11\% dos pacientes portadores de angina instável e em $9 \%$ dos portadores de IAMSST.

No seguimento clínico em seis meses, a incidência de eventos clínicos não foi diferente entre os grupos de portadores de angina instável, IAMSST e IAMCST, observando-se, respectivamente: óbito em 2\%, 4,5\% e 1,3\%; infarto do miocárdio em 1,7\%, 4,7\% e 5,1\%; nova ICP em 2,1\%, 1,6\% e 1,3\%; cirurgia cardíaca em $2,5 \%, 3,1 \%$ e $2,6 \%$; e re-hospitalização em $25 \%, 23 \%$ e $26 \%$.

A avaliação dos pacientes pelo QAS na chegada ao hospital é mostrada na Figura 1. Pacientes com angina instável apresentaram piores índices de atividade física, frequência da angina e qualidade de vida que os pacientes com IAMCST e piores índices de frequência da angina e qualidade de vida que os pacientes com IAMSST. Pacientes com IAMSST apresentaram pior índice de qualidade de vida que os pacientes com IAMCST.

A Figura 2 mostra a avaliação dos pacientes pelo QAS aos seis meses. Pacientes com angina instável ainda apresentavam piores índices de atividade física, frequência da angina e qualidade de vida que os pacientes com IAMCST, mas não houve diferença significativa quando comparados àqueles com IAMSST. Pacientes com IAMSST apresentaram piores índices de limitação física que os pacientes com IAMCST.

A Figura 3 mostra a variação dos índices do QAS obtidos na admissão hospitalar e aos seis meses de evolução. Pacientes com angina instável apresentaram maiores ganhos nos domínios da atividade física, frequência da angina e qualidade de vida que aqueles com IAMCST e maiores ganhos nos domínios de atividade física e frequência da angina que os pacientes com IAMSST. Pacientes com IAMSST e com IAMCST também demonstraram ganhos nos índices do QAS, mas as diferenças entre esses dois grupos não foram significantes.

A análise de regressão linear múltipla demonstrou que o único preditor independente de melhora da limitação física foi infarto do miocárdio [odds ratio (OR) 10,8, intervalo de confiança de 95\% (IC 95\%) 5-16,5; $\mathrm{P}<0,001]$. Os preditores independentes de melhora da frequência da angina foram angina instável (OR 10,9, IC 95\% 2,3-19,4; P = 0,01) e revascularização miocárdica (OR 15, IC 95\% 7,9-22; P < 0,001). O único preditor independente de melhora da qualidade de 
TABELA 1

Características basais da população de acordo com a síndrome coronária aguda

\begin{tabular}{|c|c|c|c|c|}
\hline Características & Angina instável $(n=246)$ & IAMSST $(n=66)$ & IAMCST (n = 79) & $\mathbf{P}$ \\
\hline Idade, anos & $61 \pm 11,5$ & $61,9 \pm 11,5$ & $58,8 \pm 11,9$ & 0,22 \\
\hline Sexo masculino, \% & 57 & 68 & 78 & 0,002 \\
\hline Raça branca, \% & 86 & 86 & 94 & 0,48 \\
\hline Hipertensão, \% & 82 & 64 & 72 & 0,003 \\
\hline Tabagismo, \% & 21 & 24 & 33 & 0,12 \\
\hline Dislipidemia, \% & 29 & 24 & 29 & 0,71 \\
\hline Diabetes melito, \% & 21 & 12 & 13 & 0,09 \\
\hline \multicolumn{5}{|l|}{ História médica, \% } \\
\hline CRM & 12 & 7.6 & 10 & 0,61 \\
\hline ICP & 34 & 23 & 11 & $<0,0001$ \\
\hline ICC & 7.3 & 3.0 & 1.3 & 0,08 \\
\hline IAM & 47 & 32 & 21 & $<0,0001$ \\
\hline Atividade física, \% & 20 & 24 & 24 & 0,93 \\
\hline $\mathrm{IMC}, \mathrm{kg} / \mathrm{m}^{2}$ & $27,5 \pm 4,5$ & $28,1 \pm 4,8$ & $27,4 \pm 4,3$ & 0,65 \\
\hline PA sistólica, mmHg & $133,2 \pm 26,5$ & $128 \pm 25,5$ & $129,2 \pm 27,8$ & 0,25 \\
\hline PA diastólica, mmHg & $76,8 \pm 15,6$ & $75,5 \pm 13,9$ & $79,9 \pm 18,8$ & 0,20 \\
\hline
\end{tabular}

CRM = cirurgia de revascularização miocárdica; IAM = infarto agudo do miocárdio; IAMCST = infarto agudo do miocárdio com elevação do segmento ST; IAMSST = infarto agudo do miocárdio sem elevação do segmento ST; ICC = insuficiência cardíaca congestiva; $\mathrm{ICP}=$ intervenção coronária percutânea; IMC = índice de massa corporal; $\mathrm{n}=$ número de pacientes; PA = pressão arterial.

TABELA 2

Tratamento medicamentoso de acordo com a síndrome coronária aguda apresentada

\begin{tabular}{|c|c|c|c|c|}
\hline Característica & Angina instável ( $n=246$ ) & IAMSST $(n=66)$ & IAMCST $(\mathbf{n}=79)$ & $\mathbf{P}$ \\
\hline \multicolumn{5}{|l|}{ Admissão } \\
\hline AAS & 92 & 95 & 97 & 0,17 \\
\hline Heparina & 72 & 82 & 71 & 0,23 \\
\hline HBPM & 23 & 24 & 24 & 0,93 \\
\hline Betabloqueadores & 85 & 89 & 80 & 0,27 \\
\hline Inibidores da ECA & 51 & 61 & 73 & 0,003 \\
\hline Estatinas & 29 & 41 & 75 & $<0,0001$ \\
\hline Nitratos EV & 29 & 35 & 29 & 0,69 \\
\hline \multicolumn{5}{|l|}{ Alta } \\
\hline AAS & 88 & 91 & 95 & 0,18 \\
\hline Betabloqueadores & 84 & 89 & 90 & 0,27 \\
\hline Inibidores da ECA & 55 & 65 & 76 & 0,003 \\
\hline Estatinas & 64 & 73 & 82 & 0,007 \\
\hline
\end{tabular}

AAS = ácido acetilsalicílico; $\mathrm{ECA}$ = enzima conversora de angiotensina; $\mathrm{EV}=$ endovenosos; $\mathrm{HBPM}=$ heparina de baixo peso molecular; IAMCST = infarto agudo do miocárdio com elevação do segmento ST; IAMSST = infarto agudo do miocárdio sem elevação do segmento ST; $\mathrm{n}$ = número de pacientes.

vida foi revascularização miocárdica (OR 9,8, IC 95\% 4,2-15,3; $\mathrm{P}<0,001)$.

\section{DISCUSSÃO}

A SCA compreende um espectro de condições que variam do IAMCST a angina instável, passando pelo
IAMSST. Neste estudo, foi analisado o estado da saúde avaliado pelo QAS na admissão hospitalar e aos seis meses de seguimento, em cada uma dessas três apresentações clínicas. Embora existam várias avaliações sobre medidas do estado da saúde em pacientes com DAC estável, as investigações clínicas em pacientes com SCA são escassas. Os dados disponíveis são geral- 


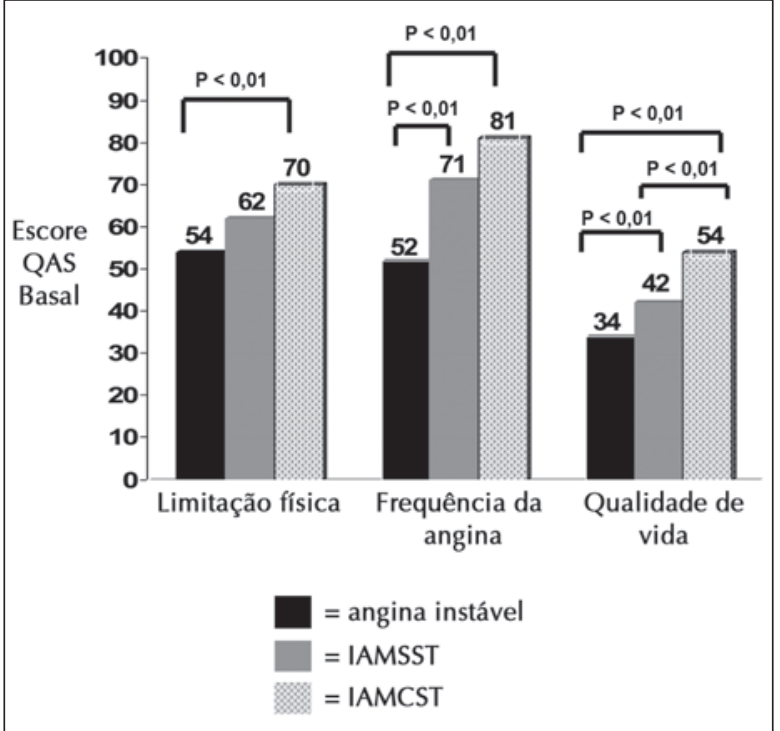

Figura 1 - Avaliação dos pacientes pelo QAS na chegada ao hospital. IAMCST = infarto agudo do miocárdio com elevação do segmento ST; IAMSST = infarto agudo do miocárdio sem elevação do segmento ST; QAS = Questionário de Angina de Seattle.

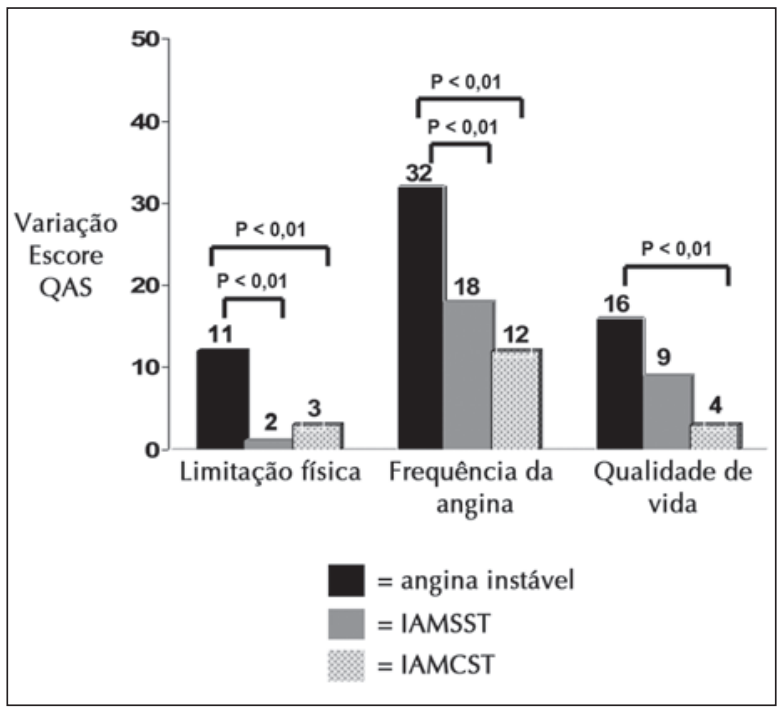

Figura 3 - Variação dos índices do QAS obtidos na admissão e aos seis meses de evolução. IAMCST = infarto agudo do miocárdio com elevação do segmento ST; IAMSST = infarto agudo do miocárdio sem elevação do segmento ST; QAS = Questionário de Angina de Seattle.

mente derivados de ensaios clínicos randomizados e podem, portanto, não representar a população não selecionada da prática clínica diária.

Neste estudo, demonstrou-se que existem diferenças significativas quando são investigados pacientes com angina instável, IAMSST e IAMCST por meio do QAS, o que desafia o conceito atual de que pacientes que apresentam um episódio de SCA sem elevação do segmento ST podem ser vistos como um grupo homogê-



Figura 2 - Avaliação dos pacientes pelo QAS aos seis meses. IAMCST = infarto agudo do miocárdio com elevação do segmento ST; IAMSST = infarto agudo do miocárdio sem elevação do segmento ST; QAS = Questionário de Angina de Seattle.

neo. Até onde se sabe, este estudo está entre os primeiros a abordar especificamente diferenças potenciais entre apresentações clínicas em pacientes com SCA por meio de uma avaliação com o QAS. Quando comparados a pacientes com IAMCST e IAMSST, aqueles com angina instável apresentam maior limitação física, mais angina e pior qualidade de vida na admissão hospitalar. Após seis meses de seguimento, embora atenuadas, essas diferenças ainda estão presentes em alguns índices. Entretanto, quando comparados com os outros grupos de pacientes, aqueles com angina instável demonstraram maior melhora em todos esses índices após seis meses de seguimento, e a análise multivariada demonstrou que o diagnóstico de angina instável na admissão hospitalar foi preditor independente de melhora da frequência da angina, aos seis meses de seguimento. Esse é um item importante de informação para os cardiologistas clínicos e para estudos futuros com medida do estado da saúde em pacientes com SCA.

Para analisar esses resultados sob uma perspectiva ampla, é preciso esclarecer por que os pacientes com angina instável apresentaram piores índices do QAS que os outros grupos. Angina instável e IAMSST são considerados quadros clínicos semelhantes, que diferem primariamente quanto ao fato de a isquemia ser grave para causar dano miocárdico suficiente para liberar marcadores de necrose. ${ }^{13}$ As SCAs compartiIham um substrato anatômico comum, ruptura ou erosão de placa aterosclerótica, com graus diferentes de trombose superimposta, o que resultará em consequências clínicas diferentes. De acordo com esse raciocínio, a angina instável é uma forma mais leve desse espectro, 
sendo lógico presumir que os índices do QAS reflitam essas diferenças, com os pacientes portadores de angina instável apresentando os melhores índices do estado da saúde. Uma possível explicação para os achados deste estudo seria que a maior frequência de fatores de risco para aterosclerose, infarto prévio do miocárdio e procedimentos de revascularização no grupo de angina instável pode ter contribuído para piorar os índices do QAS observados nesses pacientes à admissão hospitalar. Todavia, a análise multivariada demonstrou que as apresentações clínicas permaneceram como preditores independentes de melhora da frequência da angina. É também importante mencionar que o hospital onde o estudo foi realizado é um centro cardiológico terciário, com alto volume de procedimentos percutâneos e cirúrgicos, que atende pacientes com perfil mais grave.

Spertus et al. ${ }^{3}$ criaram o QAS para avaliar especificamente a DAC, porque questionários genéricos do estado da saúde não eram suficientemente eficientes para detectar alterações clínicas importantes nesses pacientes. O uso do QAS nesse contexto já foi extensamente investigado em diversos estudos, que também validaram versões traduzidas do QAS em outros países. ${ }^{22-24}$ O QAS também foi previamente validado no Brasil em uma população contemporânea de pacientes com DAC e também em pacientes tratados por ICP. ${ }^{25}$ Após ICP, demonstrou-se que a frequência da angina pré-procedimento é o indicador prognóstico mais importante de melhora da qualidade de vida. ${ }^{12}$ Enquanto benefícios substanciais relacionados à qualidade de vida são atingidos na maioria dos pacientes com angina pré-procedimento, os benefícios e riscos potenciais do procedimento em pacientes assintomáticos devem ser considerados mais cuidadosamente.

O QAS trouxe novas revelações em diversas populações de ensaios clínicos. Por exemplo, Kim et al. ${ }^{15}$ analisaram, quanto ao estado de saúde, 1.810 pacientes com SCA incluídos no ensaio randomizado Third Randomized Intervention trial of Unstable Angina (RITA3), e Janzon et al. ${ }^{16}$ estudaram uma população de 2.457 pacientes no estudo Fragmin and Fast Revascularization during Instability in Coronary Artery Disease (FRISC II). Esses autores encontraram melhores índices de saúde na estratégia de intervenção precoce, em comparação com o tratamento conservador, mas essas diferenças foram atenuadas no seguimento em um ano. As razões para explicar esses achados podem estar relacionadas às reintervenções por reestenose coronária na intervenção precoce ou ao fato de os pacientes do tratamento conservador terem necessitado revascularização miocárdica durante o seguimento. Esses resultados estão de acordo com outros estudos que demonstraram melhora do estado funcional nos pacientes submetidos a tratamento invasivo precoce. ${ }^{18}$ Outro achado relevante desses estudos foi que a melhora do estado de saúde foi explicada por menos sintomas da an- gina. Entretanto, diferenças quanto aos índices do estado da saúde, de acordo com a apresentação da SCA, não foram avaliadas por nenhum instrumento.

Em um estudo, identificou-se que diagnóstico de angina instável na alta hospitalar (ao invés de infarto do miocárdio) foi associado a menor satisfação do paciente, mas outras comparações usando o QAS não foram relatadas. ${ }^{26} \mathrm{Em}$ um registro prospectivo de 1.159 pacientes consecutivos com SCA, os pacientes de raça negra apresentavam mais angina, pior qualidade de vida e pior funcionalidade física um ano após SCA que os de raça branca. ${ }^{17}$ Também não foi apresentada uma análise dos índices do estado da saúde, de acordo com a apresentação da SCA.

\section{Limitações do estudo}

Este estudo tem algumas limitações. Por ocasião de sua realização, os níveis da troponina sérica não eram medidos rotineiramente na instituição em que o estudo foi realizado, o que poderia ter introduzido alguma distorção na definição dos grupos estudados. No entanto, os picos de CK-MB foram duas vezes maiores nos pacientes com IAMCST que nos com IAMSST e quatro vezes maiores nos pacientes com IAMSST que naqueles com angina instável, o que fala a favor de diferenças clinicamente significantes entre os grupos estudados. Além disso, mesmo que medições rotineiras dos níveis de troponina pudessem reclassificar para IAMSST pacientes com angina instável e CK-MB normal, esses pacientes normalmente apresentam elevação pequena de troponina, o que tem fraca associação com prognóstico e pior estado clínico. Por não haver um ponto exato para caracterizar a melhora dos índices dos domínios do QAS, variações positivas foram consideradas como melhora e variações negativas dos índices como piora da limitação física, da frequência da angina e da qualidade de vida. As características dos pacientes sem seguimento clínico não foram comparadas com toda a população estudada, mas apenas $5 \%$ dos pacientes inicialmente abordados foram perdidos no seguimento, número menor que o observado em estudos anteriores com o QAS. Os pacientes que morreram durante o acompanhamento foram excluídos da análise e os resultados apresentados devem ser interpretados como representativos dos que sobreviveram seis meses pós-SCA. Como a inclusão dos pacientes foi realizada há alguns anos, os avanços da farmacologia e do tratamento percutâneo nestes últimos anos também podem limitar a extrapolação desses resultados para a prática clínica atual.

\section{CONCLUSÕES}

Pacientes com angina instável apresentam piores índices de estado de saúde avaliado pelo QAS quando comparados aos pacientes com IAMSST e IAMCST, mas, por outro lado, foram os que apresentaram o maior ganho em cada um dos domínios avaliados pelo QAS. 


\section{CONFLITO DE INTERESSES}

Os autores declaram não haver conflito de interesses relacionado a este manuscrito.

\section{REFERÊNCIAS}

1. Rumsfeld JS. Health status and clinical practice: when will they meet? Circulation. 2002;106(1):5-7.

2. Souza EN, Quadros AS, Maestri R, Albarrán C, SarmentoLeite R. Preditores de mudança na qualidade de vida após um evento coronariano agudo. Arq Bras Cardiol. 2008;91(4): 252-9.

3. Spertus J, Winder JA, Dewhurst TA, Deyo RA, Prodzinski J, McDonell M, et al. Development and evaluation of the Seattle Angina Questionnaire: a new functional status measure for coronary artery disease. J Am Coll Cardiol. 1995;25(2):333-41.

4. Spertus JA, Winder J, Dewhurst TA, Deyo RA, Fihn SD. Monitoring the quality of life in patients with coronary artery disease. Am J Cardiol. 1994;74(12):1240-4.

5. Dempster M, Donnelly M. Measuring the health related quality of life of people with ischemic heart disease. Heart. 2000;83(6):641-4.

6. Dougherty CM, Dewhurst T, Nichol P, Spertus J. Comparison of three quality of life instruments in stable angina pectoris: Seattle Angina Questionnaire, Short Form Health Survey (SF-36) and Quality of Life Index-Cardiac Version III. J Clin Epidemiol. 1998;51(7):569-75.

7. Conaway DG, House J, Bandt K, Hayden L, Borkon AM, Spertus JA. The elderly: health status benefits and recovery of function one year after coronary artery bypass surgery. J Am Coll Cardiol. 2003;42(8):1421-6.

8. Mozaffarian D, Bryson L, Spertus JA, McDonell MB, Fihn SD. Anginal symptoms consistently predict total mortality among outpatients with coronary disease. Am Heart J. 2003;146(6): 1015-22.

9. Norris CM, Saunders LD, Ghali WA, Brant R, Galbraith PD, Graham M, et al. APPROACH Investigators. Health-related quality of life outcomes of patients with coronary artery disease treated with cardiac surgery, percutaneous coronary intervention or medical management. Can J Cardiol. 2004; 20(12):1259-66.

10. Pocock SJ, Henderson RA, Clayton T, Lyman GH, Chamberlain DA. Quality of life after coronary angioplasty or continued medical treatment for angina: three-year follow up in the RITA-2 trial. Randomized Intervention Treatment of Angina. J Am Coll Cardiol. 2000;35(4):907-14.

11. Spertus JA, Jones $P$, McDonell M, Fan V, Fihn SD. Health status predicts long term outcome in outpatients with coronary disease. Circulation. 2002;106(1):43-9.

12. Spertus JA, Salisbury AC, Jones PG, Conaway DG, Thompson RC. Predictors of quality-of-life benefit after percutaneous coronary intervention. Circulation. 2004;110(25):3789-94.

13. Braunwald E, Antman EM, Beasley JW, Califf RM, Cheitlin MD, Hochman JS, et al. ACC/AHA 2002 Guideline for management of patients with unstable angina and non-STsegment elevation myocardial infarction - summary article: a report of the American College of Cardiology/American
Heart Association Task Force on Practice Guidelines (Committee on the Management of Patients with Unstable Angina). Circulation. 2002;106(14):1893-900.

14. Bertrand ME, Simoons ML, Fox KAA, Wallentin LC, Hamm CW, McFadden E, et al. Management of acute coronary syndromes in patients presenting without ST elevation. Eur Heart J. 2002;23(23):1809-40. Erratum in: Eur Heart J. 2003; 24(5):485.

15. Kim J, Henderson RA, Pocock SJ, Clayton T, Sculpher MJ, Fox KA. RITA-3 Trial Investigators. Health related quality of life after interventional or conservative strategy in patients with unstable angina or non-ST-segment elevation myocardial infarction: one year of the third Randomized Intervention trial of Unstable Angina (RITA-3). J Am Coll Cardiol. 2005;45(2):221-8.

16. Janzon M, Levin L, Swahn E. Invasive treatment in unstable coronary artery disease promotes health-related quality of life: results from the FRISC II trial. Am Heart J. 2004;148(1): 114-21.

17. Spertus J, Safley D, Garg M, Jones P, Peterson ED. The influence of race on health status outcomes one year after an acute coronary syndrome. J Am Coll Cardiol. 2005;46(10): 1838-44.

18. Eisenberg MJ, Teng FF, Chaudhry MR, Ortiz J, Sobkowski W, Ebrahim I, et al. Impact of invasive management versus noninvasive management on functional status and quality of life following non-Q-wave myocardial infarction: a randomized clinical trial. Am Heart J. 2005;149(5):813-9.

19. Peterson P, Spertus JA, Magid DJ, Masoudi FA, Reid K, Hamman RF, et al. The impact of diabetes on one-year health status outcomes following acute coronary syndromes. BMC Cardiovasc Disord. 2006;6:41.

20. Rinfret S, Grines C, Cosgrove R. Quality of life after balloon angioplasty or stenting for acute myocardial infarction: oneyear results from the Stent-PAMI trial. J Am Coll Cardiol. 2001;38(6):1614-21.

21. Hosmer DW, Lemeshow S. Assessing the fit of the model. In: Hosmer DW, Lemeshow S, editors. Applied logistic regression. New York: John Wiley \& Sons; 1989. p. 135-75.

22. Garratt AM, Hutchinson A, Russell I. The UK version of the Seattle Angina Questionnaire (SAQ-UK): reliability, validity and responsiveness. J Clin Epidemiol. 2001;54(9):907-15.

23. Hofer S, Benzer W, Schubler G, von Steinbuchel N, Oldridge NB. Health-related quality of life in patients with coronary artery disease treated for angina: Validity and reliability of German translation of two specific questionnaires. Qual Life Res. 2003;12(2):199-212.

24. Pettersen KI, Reikvam A, Stavem K. Reliability and validity of the Norwegian translation of the Seattle Angina Questionnaire following myocardial infarction. Qual Life Res. 2005;14(3):883-9.

25. Franzen E. Avaliação da qualidade de vida em cardiopatia isquêmica: validação de instrumentos para uma população brasileira [dissertação]. Porto Alegre: Universidade Federal do Rio Grande do Sul; 2005.

26. Beinart SC, Sales AE, Spertus JA, Plomondon ME, Every NR, Rumsfeld JS. Impact of angina burden and other factors on treatment satisfaction after acute coronary syndromes. Am Heart J. 2003;146(4):646-52. 